\title{
Ankle Joint, Knee, Hip Distortion Syndrome Connected with the Using of Small Cars
}

\author{
Tomasz Karski ${ }^{1 *}$ and Jacek Karski ${ }^{2}$ \\ ${ }^{1}$ Vincent Pol University, Lublin, Poland \\ ${ }^{2}$ Medical University, Lublin, Poland
}

*Corresponding author: Tomasz Karski, Lublin, Poland

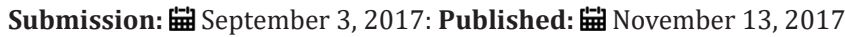

\begin{abstract} syndromes in adults can be connected with deformities such as:

a. Plano- valgus foot deformity,

b. congenital or neurological deformations of feet in children with Minimal Brain Dysfunctions (MBD),

c. Koehler's disease among girls using improper shoes.

d. insufficiency and pain of frontal part of the feet connected with limited flexion of the toes,

e. Problems of hallux valgus deformity.
\end{abstract}

The problem of hip or knee pain in adults is mostly connected with the process of arthrosis. Deformations of the feet in children and feet pain

Since some years we have observed [1] a new problem of the foot-namely "the insufficiency and pain syndrome of ankle joint". We found that this "new pain syndrome" is connected with the way people get out of the car. Now it is clear that some cases of knee and hip pain are also connected with the way people get out of the car. It is more frequent among patients who use small cars on daily basis. The way people get out of the car placing first one leg on the ground which causes the rotation movement of the whole body, distorts the ankle joint, knee joint and the hip (if there was a primary limited rotation movement in the hip because of arthrosis). This is when arthrosis processes is just begun in hips. The problem of left ankle joint, left knee and left arthrosis hip is among drivers and on the right side among passengers - in countries with "right rules traffic".

Keywords: Chronic distortion of the knee; Ankle joint; Chronic pain syndrome of hip; Knee; Ankle joint

\section{Introduction}

According to our observations, the main reason for musculoskeletal disorders, deformations and pains originate in the shortenings (in orthopedic language-contractures) of ligaments, tendons, muscles, capsules and not because of weak muscles as orthopedic surgeons and physiotherapists claim.

Various shortenings of soft tissues are the cause of the asymmetric growth and deformation among children. In adults these "shortenings" cause a disturbance of the function and different types of pain. The first article about "the shortenings of soft tissues" was presented by Professor Hans Mau in many articles. In German these "shortenings" (contractures) are called "Siebenersyndrom" $[1,2]$.

In the presented article the authors inform about the "syndromes of pain in ankle joints, knees and hips". The pathology is connected with rotation movement of the body while getting out from the small cars, repeated frequently every day.

\section{Material and Methods}

In the period of 16 years we have treated 26 patients $(n=26)$ with chronic insufficiency caused by the distortion of the ankle joint leading to limitation of dorsal flexion of the foot and pain syndrome. We also treated many patients (circa 50) suffering from knee and hip pain originating in the way they were getting out of their small cars.

The causes of the distortion of the ankle joint and knee joint as well "primary arthrosis hip joint" is in the moment of getting out of the car. This pathology is seen in the left knee joint, left ankle joint of car drivers or on the right knee and right ankle joints of passengers. It happens in countries where traffic rules drives on the right. This new medical "syndrome" has not been described until. Till now doctors interpret the walking difficulty, the pain in knee and ankle joint and the swelling of the soft tissues mostly as a peripheral vascular disease. 


\section{Hip}

While we get out into the car we need to make a rotation movement of the body, sometimes 90 degree. When the hip joint does not present any pathology we have full rotation movement such movement is easy and causes no pain. When arthrosis of the hip joint has begun - and the movements become limited, mostly the internal rotation, the patient feels pain and it becomes difficult to get out and into the car. Consequently, the occurrence of the pain while performing these movements indicates the beginning of the arthrosis of the hip. In clinical examination of arthrosis hips we can notice the limitation of the abduction, extension and most importantly, the internal rotation movement. This movement is necessary for our walking. Thus, it is not only the way we get into and out of the car but also our manner of walking, especially in "a rushed, quick walk" that patients feel pain. The first line therapy should focus on the restoration of the movements. The problem is further discussed in the following chapter.

\section{Knee}

A healthy knee joint has two-directional movement: extension and flexion. The problem appears while getting out of the car onto one leg-the left in the case of the driver and the right in the case of the passenger. While performing this movement there appears the rotation movement of the knee and it is a movement with distortion of the knee. After repeated over years this maneuver on examination we see - such knee shows signs of instability caused by loosening of lateral and medial ligaments or/and the curiae ligament of the effected joint. The problems of the knee are especially serious when varus or valgus deformity as the additional pathological changes of the axis of knee also appears. This deformation-varus or valgus - of the axis of leg, often presents since childhood and is the cause of the primary loosening of the lateral and medial ligament. The problem with the left knee is seen in drivers and the right knee in passengers of the countries where the traffic rules drives on the right. These pathological symptoms are particularly common among patients who have been driving, thus getting into and out of their cars on a daily basis over many years. Such causes of knee insufficiency reach up to about $30 \%$ of all cases among out patients.

\section{Childhood and adulthood feet problems}

The following problems can be observed:

a. flat foot deformation in children and teenagers [3-10].

b. limited plantar flexion of the toes, in adults detected by flexion toe test [10-14].

c. bunion (halluces valgi) is very frequent among women [15].

d. foot insufficiency in Koehler's disease.

e. hereditary and neurogenic disorders of the foot in children and in adults-pes valgus or pes planus- valgus residuals.

f. Since some years we have also been observing an additional problem of ankle joint-namely insufficiency connected with the improper way of getting out of the car.
Available literature discusses cases of instability of the ankle joint but regardless of the causes such as described in the present article $[16,17]$. The authors describe the syndrome present among drivers using mostly small cars who suffer from a limited movement of dorsal flexion of the foot, pain, swelling, gait restriction. The cause of these clinical symptoms lies in the permanent distortion of the ankle joint-60 to 90 degrees. The clinical examination showed loosening of ankle joint, swelling of the soft tissues especially in Achilles tendon region and in sinus tarsi.

Description of the ankle joint and knee distortion (Figure 1a1c). There are three main conditions causing problem in the region of the ankle joint.

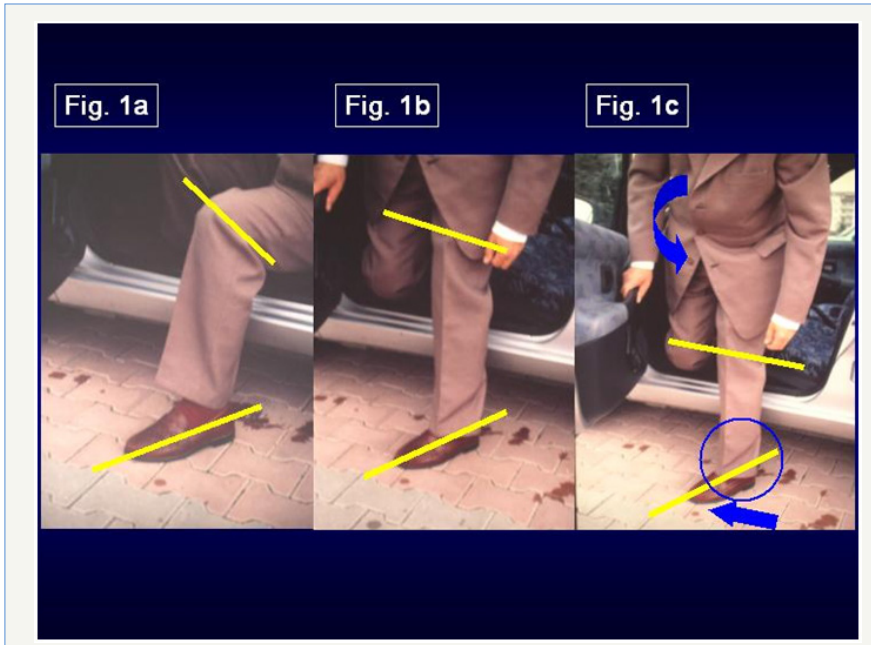

Figure 1: 1a, 1b, 1c Permanent, repeated distortions of ankle joint and knee during getting out from the small car. Explanation: foot fixed on the street, rotation of shank and whole body, permanent overloading on ankle joint with stretching of syndesmosis tibiofibularis and in resultinstability of ankle joint and pain syndrome.

a. The process of distortion lasts for many months or even years.

b. It is connected with the size of the car-this happened mostly in patients driving small cars.

c. The driver or passenger gets out of the car placing one leg on the ground and with the rotation movement of the whole body taking place on this one leg.

While getting out of the car, after opening the car door the driver puts the left leg on the ground first and next puts the whole body on the left leg, making the extensive rotation movement 6090 degrees of the body with the load of the whole body on the foot situated on the ground. This causes the distortion of the ankle joint and the knee. In this moment the ankle joint is in dorsal flexion of $0^{\circ}-5^{\circ}-10^{\circ}$ and fully stabilized. In this position, when talus between both malleoli is situated firmly there comes the rotation movement and the distortion of the ankle joint and the knee. Such repeated distortion of the ankle joint and knee over weeks, months and years causes loosening of tibio-fibular syndesmosis and consequently the instability of this joint appears. 
What is more-the stability of knee is diminished. In examination of the stability of the left ankle joint (of the drivers) or right ankle joint (of the passengers) the authors have observed the loosening even among patients with temporarily healthy functioning of the foot. The same symptoms were presented in the knee.

Clinically, we have noticed a swelling of the region of ankle joint, the knee, and the sinus tarsi and in Achilles tendon region. Patients trying to avoid the pain compensate by starting to walk on tip toes (in pes equines position). Consequently, the overstress in the triceps surae and in Achilles tendon appears as well as pain in m. triceps surae and Achilles region. Before being examined by the authors, the patients had been treated for vascular disease. There were prescribed anticoagulant medication which gave no positive effects.

\section{Treatment and prophylaxis}

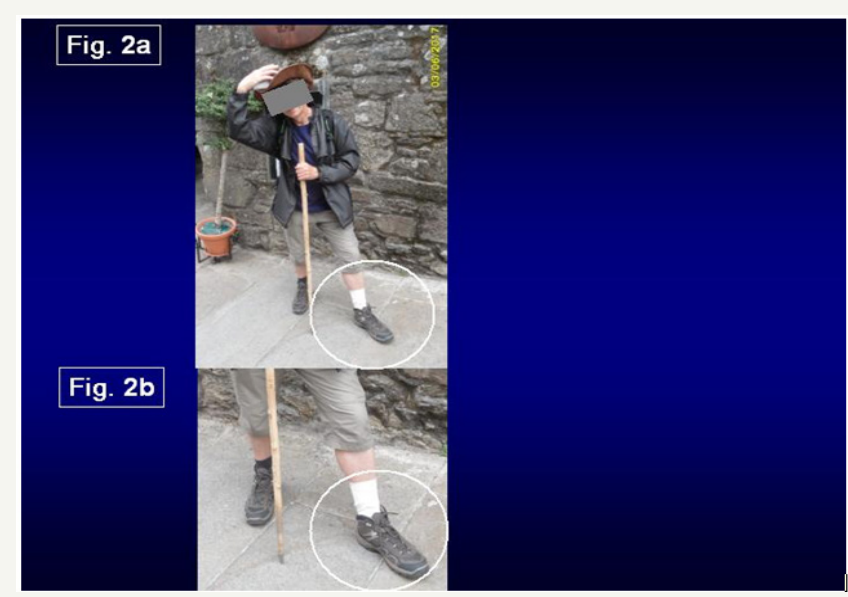

Figure 2: $2 \mathrm{a}, 2 \mathrm{~b}$ This patient $\mathrm{I}$ met in Santiago de Compostable, Spain on 2nd June 2017. Patient from Czech Republic. He told me-left foot is swollen, painful. He is unable to normal walk. He must go in equines position of the foot with help of stock-then the pain is smaller. He drives small car-Skoda. I recommended him to go out from the car in proper way-on both legs / on both feet and make exercises of the foot. He write me Email in August 2017-the swelling is away, no more pain, normal walking.

In the case of all our patients, the hip joints, knees and ankle joints were examined and the problems were thoroughly discussed. We paid particular attention to the possible coxarthrosis. We informed the patients about the need to avoid rotation movement while getting into and out of the car and while performing other daily activities involving similar rotation movement of the body. For therapy we recommended getting out of the car onto both legs and both feet (Figure 2a \& 2b). We also stressed the importance of exercises such as extension isometric exercises for knee as well dorsal and plantar flexion of the foot. Additionally, we suggested the highly beneficial role of laser, biodynamic, iontophoresis, and local criotherapy. The significance of prophylactics among all drivers all around the world was highlighted.

\section{Discussion}

The complaints concerning pain in one knee and in one foot should be analyzed also in the context of driving small cars and the manner of getting out of the cars (Figure $3 \& 4$ ). The problem appeared less frequently in the hip-only in those patients who had suffered from a limited rotation movement caused by arthrosis. In all examined cases, we observed knee instability caused by the loosening of lateral, medial or/and cruciatum ligaments, and instability in ankle joint. Interestingly, the ankle joint in dorsal flexion 0 -5-10 degree is very sensible for distortion. The anatomy and structure of ankle joint is very characteristic, that is the trochlea tali has a bigger diameter in the front part than in back part. Such anatomy enables blockage of dorsal flexion of the foot which provides stabilization of the ankle joint while taking every step, for example while walking upstairs.

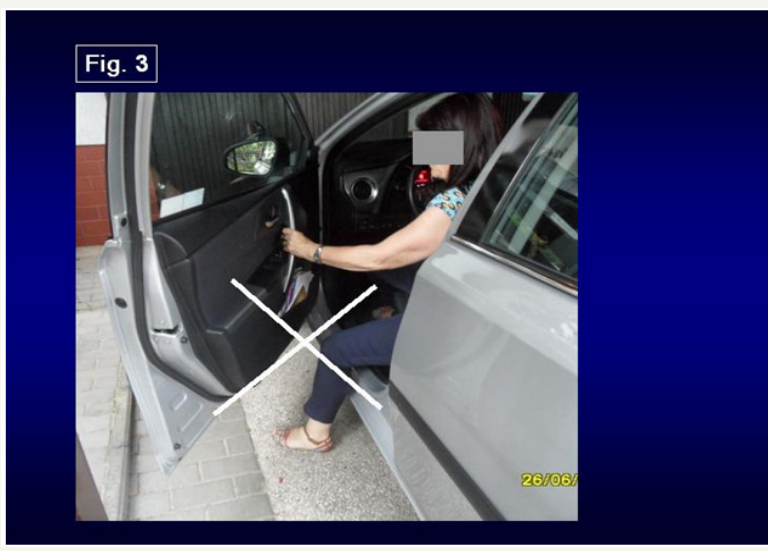

Figure 3: Not correct getting out from the car. Going on one leg, one foot makes distortion of the left ankle joint and left knee joint.

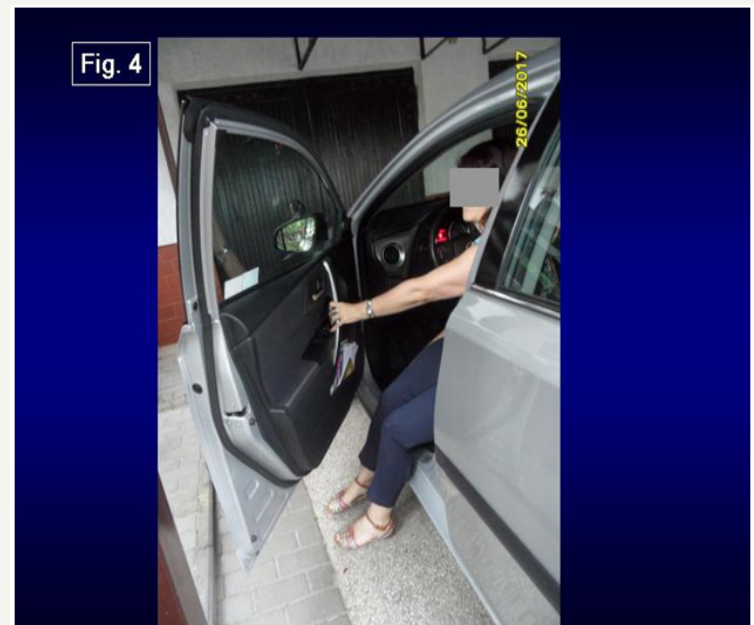

Figure 4: Proper getting out from the car. Going out on both legs, on both feet protect before distortion of the left knee and the left ankle joint.

\section{Conclusion}

We described a new pathology syndrome of the knee, ankle joint, and sometimes the hips - the chronic distortion caused by rotation movement produced while getting into (hip) and out of the car (knee, ankle joint). The problem affects the left foot and left knee of drivers and the right foot and the right knee of passengers of countries where the traffic rules drives on the right. 
For therapy we recommend a change in the manner of getting out of the car that is both legs and both feet without any rotation movement of the trunk. Additionally, we advice kinesiotherapy, hydro massage, laser, biodynamic therapy, All orthopedic surgeons, rehabilitation doctors and physiotherapists should be introduced to this problem and they should further inform about necessity of prophylactics in all countries.

\section{Acknowledgement}

Many thanks for correcting of the English to Miss Honorata Menet and Dr Katarzyna Karska.

\section{References}

1. Karski J, Tomasz K (2014) Syndrome of contractures and deformities according to Prof. Hans Mau as the primary cause of motoric deformities in children. Case studies including deformities of hips, neck, shank and spine. Arch Physiother Glob Res 18 (2): 15-23.

2. Karski T, Karski J (2015) Syndrome of Contractures and Deformities according to Prof. Hans Mau as Primary Cause of Hip, Neck, Shank and Spine Deformities in Babies, Youth and Adults. American Research Journal of Medicine and Surgery 1(2).

3. Karski T (1990) Congenital and acquired foot defects in children, Orthopedics, traumatology and rehabilitation of the organs of motion. In: St Piatkowski (Eds.), PZWL, Warsaw, 217-220.

4. Karski T, Konera W, Malicki M (1990) Static and jatrogenic knee plate deformities in children. Explanation of the phenomena and possibilities of therapy. Szekesfehervar, October, 4-6, Orthopedic Congress in Hungary. Dni Ortopedyczne Węgierskiego Towarzystwa Ortopedycznego.

5. Karski T (1990) Activity of the Lublin Podiatrist Orthopedic Clinic Under the direction of Prof. St. Piatkowski. Chir. Movement Tool and Ortop Polska LV, 3, 179-181.

6. Karski T, Karski J, Snela S, Ostrowski J (1995) Crease foot deformities in children with spastic shortening of the Achilles tendon. International Community Congress, Kurzfassungen-Block 6, Berlin.
7. Karski J, Karski T, Snela S, Ostrowski J Foot injuries in children with spastic shortening of the Achilles tendon. Orthopädieschuhtechnik 9/96, Journal of Prevention and Rehabilitation, 24-26.

8. Karski T, Frelek KM, Papierkowski A, Taczała J (1996) Foot deformities with children with cerebral palsy Annual Meeting of the Hungarian Orthopedic Association, Nyiregyhaza 20-22.

9. Karski J (1995) Pes valgus deformities in children with spastic shortening of the Achilles tendon, International Gemeinschaftskongress, Berlin, 266-267.

10. www.ortopedia.karski.lublin.pl

11. Karski $T$ (1971) The usefulness of the toes flexions test in toes assessment of foot insufficiency. Surgery of the organs of movement and orthopedics Poland, 36: 6.

12. Karski T (1975) Separation of construction and activities of sensitive feet. Leather review No. 6/348, 18.

13. Karski T (1980) Tests useful in assessing foot hazards, biomechanics and prevention of static foot distortion. Materials from the Scientific Session. Lublin, 1979, PZWL 4-5 (12): 113-116.

14. Karski $\mathrm{T}$ (1985) The toe flexion test to detect early stages of functional disorders and deformities of the forefoot. Beitr Orthop Traumat 32: 305307.

15. Menz HB (2016) Chronic foot pain in older people. Maturitas. 91: 110114.

16. Hernández AM, Mellado RM, Páramo DP, García LL, Vilà RJ (2017) Chronic ankle instability: Arthroscopic anatomical repair. Rev Esp Cir Ortop Traumatol 61(2): 104-110.

17. Hurst B, Branthwaite H, Greenhalgh A, Chockalingam N (2017) Medicalgrade footwear: the impact of fit and comfort. J Foot Ankle Research 10: 